\title{
Self-organized Pattern Formation of Whole-body Action-perception Coordination: A Study of Street Dancers and Non-dancers
}

\author{
Akito Miura $^{* \dagger} \quad$ Kazutoshi Kudo ${ }^{*}$ Kimitaka Nakazawa ${ }^{*}$ \\ (*)Graduate School of Arts and Sciences, The University of Tokyo, Japan \\ (†)Japan Society for the Promotion of Science, Japan \\ E-mail:akito.miura@gmail.com,kudo@idaten.c.u-tokyo.ac.jp,nakazawa@idaten.c.u-tokyo.ac.jp
}

\begin{abstract}
The aim of this study was to investigate whether whole-body action-perception coordination is governed by dynamical principles using basic street dance movement. Six skilled street dancers and 8 novice controls performed 2 movement patterns: knee-flexion-on-the-beat (down movement) and knee-extension-on-the-beat (up movement) in the standing position, and they did not intervene in the pattern change. The beat rate increased/decreased between 60 and 220 beats per minute (bpm) in steps of $20 \mathrm{bpm}$. The relative phase between knee movements (as measured by twin-axis electrogoniometer) and the beat were calculated. In the ascending beat rate condition of the up movement, phase transition from knee-extension-on-the-beat to knee-flexion-on-the-beat occurred at averages of $125 \mathrm{bpm}$ in non-dancers and $164 \mathrm{bpm}$ in dancers. Critical fluctuation and hysteresis were also observed. These results suggest that whole-body action-perception pattern formation is governed by general dynamical principles and that critical frequency could be a parameter of proficiency in street dance.
\end{abstract}

\section{Introduction}

Numerous studies have suggested that dynamical principles govern movement coordination in humans. This has been investigated in terms of phase transition paradigms. In Kelso's experiment [1], when participants were instructed to perform adduction-abduction movements with the index fingers of both hands in an anti-phase pattern (homologous muscle groups contracting in an alternating fashion) at driving frequencies specified by a metronome, the movement pattern was suddenly changed to an intrinsically stable pattern: in-phase pattern (homologous muscle groups contracting simultaneously) at a critical frequency. The phase transition to an intrinsically stable coordination pattern has been confirmed in various forms of movement coordination [2-4], and these intrinsic dynamics can be modified by motor learning [5].

Among the aforementioned research on phase transition phenomena of human movement coordination, there is some evidence that focuses on that of whole-body movement coordination in a standing position. Because many types of human activities include whole-body coordinated movement, it is important to determine whether whole-body movement is governed by general dynamical principles. Bardy et al. [3] reported that a transition between 2 distinct coordination modes (in-phase: ankle and hip joints oscillating in the same direction, and anti-phase: the 2 joints oscillating in opposite directions) occurred in accordance with the alternation of movement frequency in postural control. Miura et al. [6] reported that a knee-extension-on-the-beat pattern in a standing position, which is typically seen in street dance, was unintentionally replaced by knee-flexion-on-the-beat pattern when movement frequency specified by metronome beat was high, but skilled street dancers could resist this replacement. These studies suggest that whole-body movement in a standing position is also governed by dynamical principles and that motor learning can modify intrinsic dynamics of whole-body action-perception coordination.

In Miura et al.'s study [6], the metronome beat rate was not varied systematically or continuously within a trial, and participants were instructed to adjust the movement to the beat while in motion. Thus, other criteria associated with self-organized pattern formation, such as the critical frequency at which phase transition occurs; critical fluctuation, meaning that the variability of order parameters (i.e., relative phase angle) increases around the critical frequency; and hysteresis, which implies that phase angle profiles in ascending and descending beat rate conditions are not identical, were not observed. However, we

This is an Open Access article distributed under the terms of the Creative Commons Attribution-Noncommercial License 3.0, which permits unrestricted use, distribution, and reproduction in any noncommercial medium, provided the original work is properly cited. 
hypothesized that if we investigate this rhythmic knee-bending movement with a continuously changing metronome beat and instruct the participants not to intervene in the pattern change, hysteresis and critical fluctuation can be observed. In addition, critical frequency in street dancers could be higher than that in non-dancers if intrinsic dynamics are modified by long-term practice. Therefore, we investigated the effect of movement frequency, coordination modes, and motor learning on pattern formation of whole-body action-perception coordination by comparing skilled street dancers and non-dancers.

\section{Methods}

\subsection{Participants}

Six skilled street dancers and 8 control participants participated in this experiment. The street dancers (men, age $28.0 \pm 5.3$ years, $\mathrm{M} \pm \mathrm{SD}$ ) had $12.3 \pm 5.6$ years of dancing experience, and all of them had won celebrated national or international street dance competitions. The novice controls (men, age $25.5 \pm 2.3$ years) had no experience in street dance. Hereafter, we refer to the dancer group simply as dancers and the control group as non-dancers. Furthermore, informed consent was sought from all individuals for their participation in the experiment. This study was approved by the Ethics Committee of the Graduate School of Arts and Sciences of the University of Tokyo.

\subsection{Experimental task and procedure}

The experimental task was the performance of basic street dance movements, which consisted of bouncing up and down repeatedly by bending at the knees in synchronization to a metronome beat (see Ref. 6 for detail). There were 2 movement conditions. One was to flex the knees on the beat (down movement condition) and the other was to extend the knees on the beat (up movement condition). The participants were instructed to maintain a 1:1 relationship between movement and beat and not to intervene in the spontaneous pattern change. The beat rate increased/decreased between 60 and 220 beats per minute (bpm) in steps of $20 \mathrm{bpm}(9$ frequency plateaus). Each frequency plateau had 16 beats. Three sets of 4 conditions ( 2 movements and 2 beat rate conditions) were performed in a pseudo-random order.

\subsection{Data collection and analysis}

Knee angular displacement was measured using a twin-axis electrogoniometer placed over the right knee joint. Knee angular velocity was obtained by differentiating angular displacement. The phase angle of the beat time on the phase plane was calculated. First, the knee angular displacement and angular velocity were converted to a $Z$-value and plotted on the phase plane. The beat onset time was then superposed on the phase plane trajectory. The phase angle of beat time and standard deviation (SD) of the phase angle were calculated using circular statistics [7].

\section{Results}

Figure 1 illustrates typical examples of the phase angle of beat time for 1 dancer. In the ascending beat rate of the up movement condition, phase transition from the up movement (knee-extension-on-the-beat) to the down movement (knee-flexion-on-the-beat) occurred (Fig. 1b). Around the frequency at which phase transition occurred, SD of the relative phase between movement and beat was enhanced; that is, critical fluctuation was observed. In the ascending beat rate of the down movement condition, no phase transition occurred (Fig. 1a).

Figure 2 shows the histograms of the phase angle of 16 cycles at each frequency of all 3 sets of all dancers. In both the ascending and descending beat rates of the down movement conditions, no phase transition occurred (Fig. 2a,b). On the contrary, in the ascending beat rate of the up movement condition, phase transition from the up movement (knee-extension-on-the-beat) to the down movement (knee-flexion-on-the-beat) occurred (Fig. 2c). In the descending beat rate of the up movement condition, dancers performed the down movement instead of the up movement at a high movement frequency, and as the beat rate slowed, some participants showed transition to the up movement while others showed no such transition (Fig. 2d). In other words, hysteresis was observed. Non-dancers showed a similar pattern of the phase angle.

We calculated the critical frequency at which phase transition occurred. Prior to the calculation, we defined the average of all phase angles of all 3 sets of all participants under down movement conditions of both beat rate conditions $\pm 90^{\circ}$ as the down movement range because there was no phase transition under the down movement conditions. We defined the critical frequency as the beat rate at which 3 consecutive cycles deviated from the down movement range. Dancers showed a significantly higher critical frequency (average of $164 \mathrm{bpm}$ ) than non-dancers (average of $125 \mathrm{bpm}$ ) under the up movement condition of the ascending beat rate condition $(p<.05)$. 
a

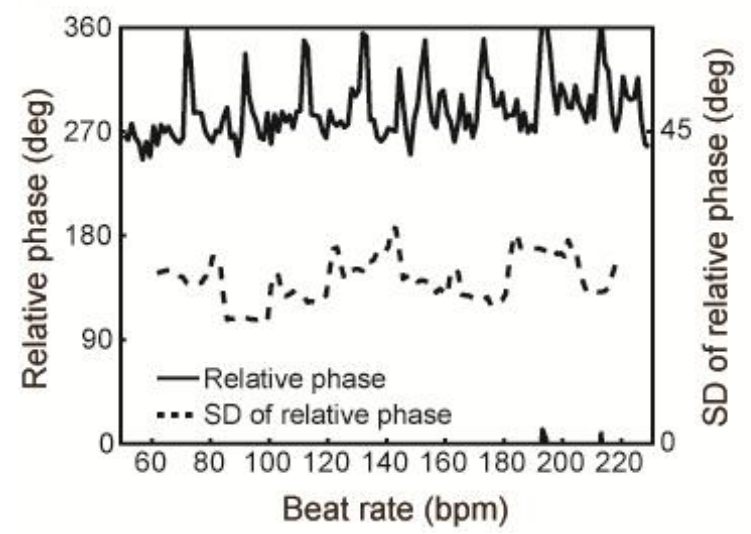

b

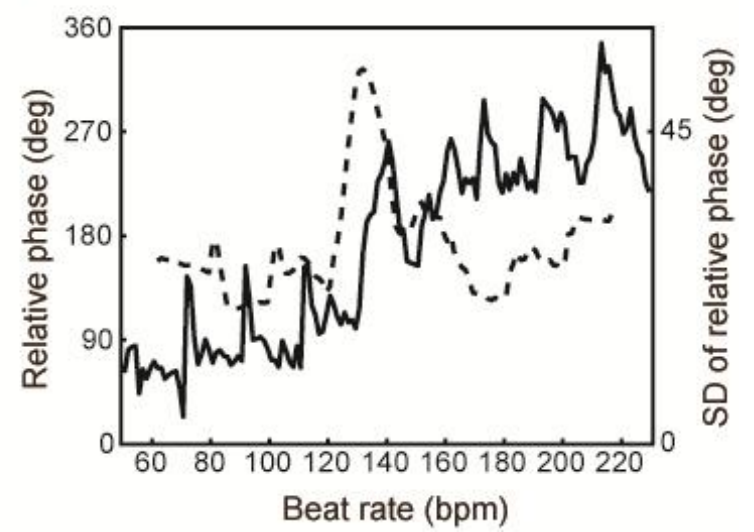

Figure 1 Typical examples of relative phase and SD of the relative phase in ascending beat rate of (a) down movement and (b) up movement conditions.

a

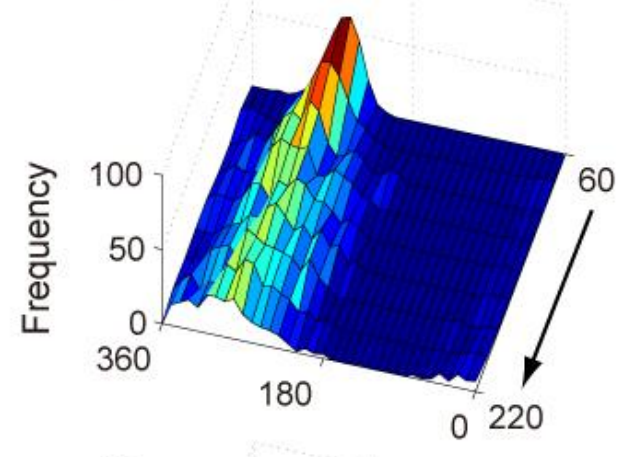

C

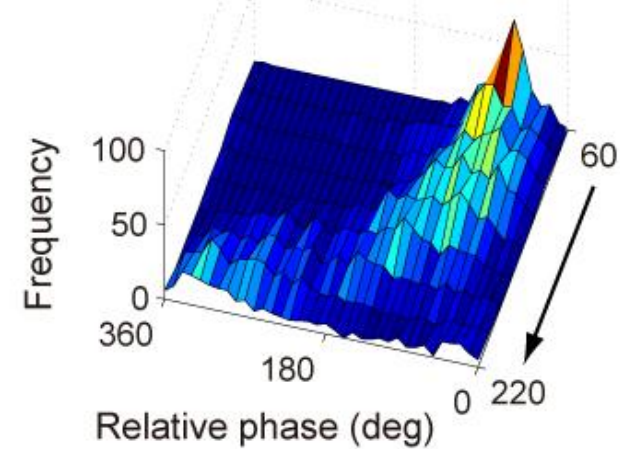

b

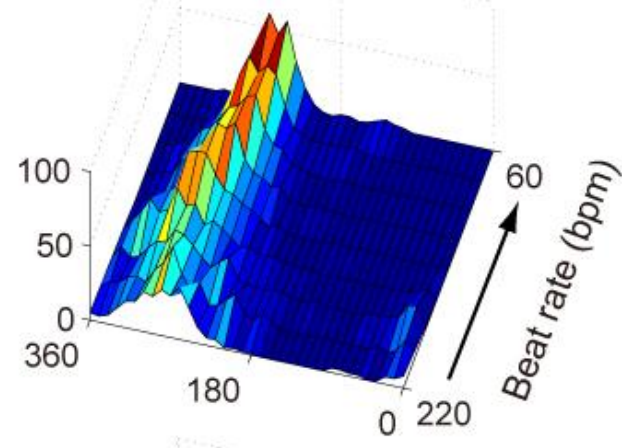

d

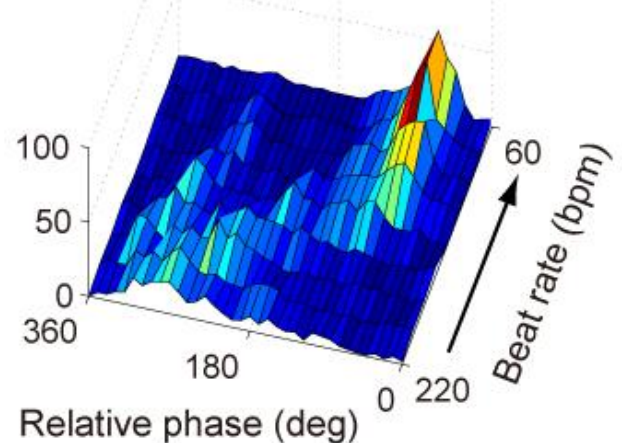

Figure 2 Histograms of the phase angle of dancers in ascending beat rate of (a) down movement and (c) up movement conditions, and in descending beat rate of (b) down movement and (d) up movement conditions.

\section{Discussion}

This study investigated whether pattern formation of whole-body action-perception coordination is self-organized. Dancers and non-dancers performed 2 types of knee-bending movements in a standing position as a whole-body task at driving frequencies specified by a metronome beat. We confirmed criteria associated with self-organized pattern formation: phase transition, critical fluctuation, and hysteresis. This suggests that whole-body action-perception coordination is governed in a self-organized manner.

Previous studies have revealed that the organization of the coordination pattern is affected by various 
constraints, such as joint motion [8] and gravity [9]. Carson et al. [8] reported that the flexion-on-the-beat pattern is more stable than is the extension-on-the-beat pattern in finger action-perception coordination. In addition, Carson et al. [9] demonstrated that in wrist action-perception coordination, when the effect of gravity on the hand was reversed with a robotic system, the upward-on-the-beat pattern was more stable than the downward-on-the-beat pattern, which was explained by the economy of action that was derived from the exploitation of gravity. In the present study, phase transition from the up movement (knee-extension-on-the-beat: synchronization of movement in the opposite direction of gravity with the beat) to the down movement (knee-flexion-on-the-beat: synchronization of movement with gravity with the beat) occurred. Thus, the results of these previous studies [8, 9] are highly consistent with our results, which suggest that joint motion (flexion/extension) and movement direction relative to gravity are the salient constraints in whole-body action-perception coordination.

Zanone and Kelso [5] showed that an intrinsically unstable coordination pattern could become stable with practice. This has been confirmed in various types of coordination [6]. In this study, dancers showed a significantly higher critical frequency than did non-dancers. This result is consistent with Miura et al.'s study [6], in which skilled street dancers could resist unintentional replacement of the up movement by performing the down movement at high frequencies. These findings suggest that motor learning of street dance could be construed as overcoming intrinsic constraints imposed on spatiotemporal organization of whole-body coordinated movement [2, 10]. Moreover, these results suggest that critical frequency could be a parameter of proficiency in street dance and that critical frequency could be an effective movement frequency in training for overcoming phase transition from the up movement to the down movement, which should be investigated in future studies.

\section{Conclusion}

Our results of phase transition, critical fluctuation, and hysteresis suggest that whole-body action-perception pattern formation is governed in a self-organized manner, providing additional evidence that human movement coordination is governed by general dynamical principles. We would like to emphasize that critical frequency could be a parameter of proficiency in street dance and that a dynamical systems approach will contribute not only to understanding the process of skill acquisition but also to developing effective practice methods.

\section{Acknowledgments}

This study was partly supported by Grants-in-Aid for Scientific Research (No. 21300215 and No. 20240060) from the Japan Society for the Promotion of Science (JSPS) awarded to K. Kudo, a Grant-in-Aid for JSPS Fellows (No. 23.9480) awarded to A. Miura, and a Sports Research Grant from Yamaha Motor Foundation for Sports awarded to A. Miura and K. Kudo.

\section{References}

[1] J.A.S. Kelso. Phase-transitions and critical-behavior in human bimanual coordination, American Journal of Physiology, American Physiological Society, Bethesda, MD, USA, pages. 1000-1004, 1984

[2] S. Swinnen. Intermanual coordination: from behavioural principles to neural-network interactions, Nature Reviews Neuroscience, Nature Publishing Group, UK, pages. 348-359, 2002

[3] B.G. Bardy, O. Oullier, R.J. Bootsma, and T.A. Stoffregen. Dynamics of human postural transitions, Journal of Experimental Psychology: Human Perception and Performance, American Psychological Association, Washington, D.C., USA, pages. 499-514, 2002

[4] J.A.S. Kelso, J.D. DelColle, and G. Schoner. Action-perception as a pattern formation process, Attention and Performance XIII, Psychology Press, pages. 139-169, 1990

[5] P.G. Zanone and J.A.S. Kelso. Evolution of behavioral attractors with learning - Nonequilibrium phase-transitions. Journal of Experimental Psychology: Human Perception and Performance, American Psychological Association, Washington, D.C., USA, pages. 403-421, 1992

[6] A. Miura, K. Kudo, T. Ohtsuki, and H. Kanehisa. Coordination modes in sensorimotor synchronization of whole-body movement: A study of street dancers and non-dancers. Human Movement Science, Elsevier, Amsterdam, in press

[7] E. Batschelet. Circular Statistics in Biology, New York: Academic Press Inc., 1981.

[8] R.G. Carson. Neuromuscular-skeletal constraints upon the dynamics of perception-action coupling. Experimental Brain Research, Springer, New York, NY, USA, pages. 99-110, 1996

[9] R.G. Carson, Y. Oytam, and S. Riek. Artificial gravity reveals that economy of action determines the stability of sensorimotor coordination. PLoS one, the Public Library of Science, CA, USA, pages. e5248, 2009

[10]S. Fujii, K. Kudo, T. Ohtsuki, and S. Oda. Intrinsic constraint of asymmetry acting as a control parameter on rapid, rhythmic bimanual coordination: A study of professional drummers and non-drummers. Journal of Neurophysiology, American Physiological Society, Bethesda, MD, USA, pages. 2178-2186, 2010 